\title{
Testosterone Increases Amygdala Reactivity in Middle-Aged Women to a Young Adulthood Level
}

\author{
Guido A van Wingen*, 1,2, Staś A Zylicz', Sara Pieters', Claudia Mattern³, Robbert Jan Verkes², \\ Jan K Buitelaar ${ }^{2}$ and Guillén Fernández ${ }^{1,4}$ \\ 'FC Donders Centre for Cognitive Neuroimaging, Radboud University Nijmegen, Nijmegen, The Netherlands; '2Department of Psychiatry, \\ Radboud University Nijmegen Medical Centre, Nijmegen, The Netherlands; ${ }^{3} \mathrm{M}$ et P Pharma AG, Mühlebachstrasse, Switzerland; ${ }^{4}$ Department of \\ Neurology, Radboud University Nijmegen Medical Centre, Nijmegen, The Netherlands
}

\begin{abstract}
Testosterone modulates mood and sexual function in women. However, androgen levels decline with age, which may relate to the ageassociated change in sexual functioning and the prevalence of mood and anxiety disorders. These effects of testosterone are potentially mediated by the amygdala. In the present study, we investigated whether the age-related decline in androgen levels is associated with reduced amygdala activity, and whether exogenous testosterone can restore amygdala activity. Healthy young and middle-aged women participated during the early follicular phase of the menstrual cycle, and amygdala responses to biologically salient stimuli were measured with functional magnetic resonance imaging ( $\mathrm{fMRI}$ ). Androgen levels were lower in middle-aged than young women, which was associated with decreased amygdala reactivity. Endogenous testosterone levels correlated positively with amygdala reactivity across the young and middle-aged women. The middle-aged women received a single nasal dose of testosterone in a double-blind, placebocontrolled, crossover manner, which rapidly increased amygdala reactivity to a level comparable to the young women. The enhanced testosterone levels correlated positively with superior frontal cortex responses and negatively with orbitofrontal cortex responses across individuals, which may reflect testosterone-induced changes in amygdala regulation. These results show that testosterone modulates amygdala reactivity in women, and suggest that the age-related decline in androgen levels contribute to the decrease in amygdala reactivity.
\end{abstract}

Neuropsychopharmacology (2009) 34, 539-547; doi: I 0. I038/npp.2008.2; published online 30 January 2008

Keywords: $\mathrm{fMRl}$; testosterone; amygdala; emotion; mood; sexual function

\section{INTRODUCTION}

Androgen levels in women decline with age, such that a woman has about half her early adulthood levels at age forty (Zumoff et al, 1995; Davison et al, 2005). Synchronously with the age-related decline in androgen levels, the prevalence of affective and anxiety disorders decreases (Kessler et al, 1994, 2003; Henderson et al, 1998). However, sexual functioning also declines in women (Hayes and Dennerstein, 2005; Hayes et al, 2007). Thus, the change in androgen levels might contribute to both the susceptibility for mood disorders and for sexual dysfunction. For example, the decrease in gonadal steroid hormone concentrations after oophorectomy is associated with a worsening of mood and sexual motivation, which improves with subsequent testosterone treatment (Sherwin and Gelfand, 1985; Sherwin et al, 1985; Shifren et al, 2000). In

*Correspondence: GA van Wingen, FC Donders Centre for Cognitive Neuroimaging, Kapittelweg 29, 6525 EN Nijmegen, The Netherlands, Tel: + 31243668219 , Fax: + 31243610989 ,

E-mail: guido.vanwingen@fcdonders.ru.nl

Received 28 August 2007; revised II December 2007; accepted 20

December 2007 addition, testosterone treatment also improves well-being, mood, and sexual function in healthy premenopausal women with low libido (Goldstat et al, 2003). However, it remains unknown by which neural mechanism testosterone influences mood and sexual function, and whether this is related to the age-related decline in androgen levels.

Animal studies suggest that testosterone influences mood and sexual function by modulating amygdala activity, a brain structure implicated in mood and anxiety regulation (Drevets, 2003; Rauch et al, 2003) and sexual arousal (Karama et al, 2002; Hamann et al, 2004; Gizewski et al, 2006). Testosterone modulates functioning of amygdala neurons in male and female rodents (Kendrick and Drewett, 1979; Kendrick, 1981), and testosterone infusions into the amygdala increase sexual behavior in male rodents (Wood and Newman, 1995; Bialy and Sachs, 2002). Whereas these studies investigated the effects of prolonged testosterone exposure recent studies suggest, that testosterone influences anxiety and sexual behavior also rapidly (Aikey et al, 2002; James and Nyby, 2002). Moreover, a single testosterone administration already increases physiological arousal in response to affective and erotic stimuli in women (Tuiten et al, 2000; van Honk et al, 2001). 
This study investigated whether nasally applied testosterone rapidly increases amygdala reactivity in healthy, naturally cycling, middle-aged women, using a doubleblind, placebo-controlled, crossover design. Amygdala reactivity to biologically salient stimuli was measured with functional magnetic resonance imaging. To investigate whether the diminished endogenous levels of androgens during middle adulthood influence amygdala activity, the placebo session was compared with the placebo session of young, healthy, naturally cycling women, who participated in an identical experiment with a different drug manipulation (van Wingen et al, 2007) and who were expected to have higher androgen levels.

\section{MATERIALS AND METHODS}

\section{Participants}

This study included two groups of participants. The experimental group consisted of 27 middle-aged, naturally cycling, premenopausal women. One participant was not available for the second study occasion, and data of one session of another subject were lost due to a technical failure. Therefore, the results are based on the data of the remaining twenty-five participants (mean age 42 years; range: 37-50 years). The reference group consisted of 17 young, naturally cycling women (mean age 23 years; range: 19-30 years) who participated previously in another study with the same experimental procedure but a different drug manipulation (van Wingen et al, 2007). All 44 participants were physically and mentally healthy as determined by a structured interview, were free of medication and hormonal contraceptives, and right handed. The study was approved by the local ethics committee (CMO Regio ArnhemNijmegen, The Netherlands). All participants gave written informed consent after the study had been explained to them.

\section{Design and Procedure}

All women participated two times during the early follicular phase (days 1-7) of different menstrual cycles, to ensure low endogenous hormone levels. The middle-aged women first completed a sexual arousal questionnaire (SAQ) (Tuiten et al, 2000), before the first venous blood sample was collected. Thereafter, a nasal dose of testosterone $(0.9 \mathrm{mg}$; Noseafix ${ }^{\circledR}$ ) or placebo was administered in a double-blind, crossover manner. Prior to scanning (30 min after drug intake), they completed the SAQ again and a second blood sample was drawn. About $140 \mathrm{~min}$ after drug intake, the final SAQ was administered, after which the participants completed mood (Mood Rating Scale; MRS) (Bond and Lader, 1974) and state anxiety (State Trait Anxiety Inventory; STAI) (Spielberger et al, 1970) questionnaires. The young women of the reference group completed a similar procedure, described in van Wingen et al (2007).

\section{Behavioral Task}

The experimental paradigm consisted of a blocked design, including an emotion condition, and a visuo-motor control condition. This paradigm has been used previously to investigate drug effects on amygdala reactivity (Hariri et al, 2002; Paulus et al, 2005; van Wingen et al, 2007). It robustly engages the amygdala, by contrasting the response to simultaneously present angry and fearful face stimuli (http://www.macbrain.org) with the response to ellipses (that consisted of scrambles of the same face stimuli). The results therefore do not show emotion-specific effects, but rather a general response to salient, biologically relevant stimuli.

Two emotion blocks were interleaved with three control blocks, and each $30 \mathrm{~s}$ block consisted of six $5 \mathrm{~s}$ trials. Each trial consisted of three simultaneously presented stimuli, with the cue stimulus presented above the target and distractor. In the emotion condition, an angry or fearful face was presented on top as cue, and subjects had to indicate by an appropriate button press, which of the bottom two faces (one angry and one fearful) matched the cue in emotional expression. The three simultaneously presented faces per trial were from different persons from the same sex. Half the trials presented faces of men and half of women, half of each target emotion (angry or fearful). In the control condition, a horizontally- or vertically-oriented ellipse was presented as cue above two ellipses (one vertical and one horizontal), and subjects had to select the identically oriented ellipse.

\section{Magnetic Resonance Imaging Data Acquisition}

MR data was acquired with a $1.5 \mathrm{~T}$ Siemens Sonata MR scanner (Siemens, Erlangen, Germany), equipped with a standard head coil. Seventy-six T2*-weighted blood oxygenation level-dependent (Bold) images were acquired using echo-planar imaging (EPI) with an echo time of $30 \mathrm{~ms}$ to reduce signal dropout, with each image volume consisting of 33 axial slices ( $3 \mathrm{~mm}, 0.5 \mathrm{~mm}$ slice-gap, TR $=2.290 \mathrm{~s}$, $64 \times 64$ matrix, $\mathrm{FOV}=224 \mathrm{~mm}, \mathrm{FA}=90^{\circ}$ ). In addition, a high resolution $\mathrm{T} 1$-weighted structural $\mathrm{MR}$ image was acquired for spatial normalization procedures (3D MP-RAGE, $\mathrm{TR}=2250 \mathrm{~ms}, \mathrm{TE}=3.93 \mathrm{~ms}, \mathrm{TI}=850 \mathrm{~ms}, 176$ contiguous $1 \mathrm{~mm}$ slices, $256 \times 256$ matrix, $\mathrm{FOV}=256 \mathrm{~mm}$ ).

\section{Functional Magnetic Resonance Imaging Data Analysis}

Image analysis was performed with SPM2 (Wellcome Department of Imaging Neuroscience, London, UK). The first five EPI-volumes were discarded to allow for $\mathrm{T} 1$ equilibration, and the remaining images were realigned to the first volume. Images were then corrected for differences in slice acquisition time, spatially normalized to the Montreal Neurological Institute T1 template, super-sampled into $2 \times 2 \times 2 \mathrm{~mm}^{3}$ voxels, and spatially smoothed with a Gaussian kernel of $10 \mathrm{~mm}$ FWHM.

Statistical analysis was performed within the framework of the general linear model (Friston et al, 1995). For each drug condition, the two experimental conditions were modeled as box-car regressors convolved with the canonical hemodynamic response function of SPM2. In addition, the realignment parameters were included to model potential movement artifacts and a high-pass filter (cutoff at $1 / 128 \mathrm{~Hz}$ ). To account for various global effects, the EPIdata was proportionally scaled. Temporal autocorrelation was modeled with an $\mathrm{AR}(1)$ process and the parameter estimates were obtained by maximum likelihood estimation 
(Friston et al, 2002) to allow departures from sphericity. Parameter images contrasting the emotion and visuo-motor control condition were obtained, and analyzed in subsequent random-effects analyses. Drug effects were assessed with paired $t$-tests, and independent samples $t$-tests were used to identify age effects. Statistical tests were family-wise error rate corrected for multiple comparisons across the whole brain. A small volume correction (Worsley et al, 1996) was used to correct for multiple comparisons across the search volume for regions of interest (ROI). As the basolateral amygdala is critically involved in sexual motivation (Everitt, 1990), the search volume for the amygdala was defined as a sphere with $7 \mathrm{~mm}$ radius around the probabilistic cytoarchitectonic center of the basolateral amygdala ([-26 -8 -18] and [28 -8 -18]), which approximates total amygdala volume (Amunts et al, 2005; Eickhoff et al, 2005). In addition, the face responsive region in the fusiform gyrus was defined as a sphere with $10 \mathrm{~mm}$ radius around previously reported Talairach coordinates (Kanwisher et al, 1997), that were transformed into MNI-space (http://imaging.mrc-cbu.cam.ac.uk/imaging/ MniTalairach; [ $-36-64-16]$ and [ $40-56-16])$. The inverse transformation was used to convert the results from MNI to Talairach space, and all local activation maxima are reported in Talairach coordinates. In addition, ROI analyses were conducted to compare amygdala reactivity across the different conditions, for which the mean activity within amygdala search volumes was extracted (Brett $e t$ al, 2002). Effect sizes for these ROI analyses were calculated, and $r=0.10$ indicates a small effect, $r=0.30$ a medium effect, and $r=0.50$ a large effect (Cohen, 1992).

\section{Serum Analysis}

The serum concentrations of total testosterone $(\mathrm{T})$ were measured by liquid chromatography with tandem mass spectrometry detection after non-polar solvent extraction. Serum concentrations of dehydroepiandrosterone sulfate (DHEA-S), dihydrotestosterone (DHT), and $3 \alpha$-androstanediol glucuronide ( $3 \alpha$-diol) were measured by radioimmunoassay. The coefficients of variation for $\mathrm{T}$ were $4.3-14.8 \%$ for the inter-assay and $0.7-17.3 \%$ for the intra-assay precision, for DHEA-S $6.5-8.4 \%$ for the inter-assay and 3.6-6.9\% for the intra-assay precision, for DHT $6.2-11.9 \%$ for the inter-assay and $2.2-18.1 \%$ for the intra-assay precision, and for $3 \alpha$-diol $11-24 \%$ for the inter-assay and $8.0-23 \%$ for the intra-assay precision.

\section{RESULTS}

\section{Age Effects on Androgen Levels and Amygdala Reactivity}

Serum concentrations, questionnaire, and behavioral performance. Baseline serum concentrations of dehydroepiandrosterone sulfate (DHEA-S; $t(40)=4.6, p<0.001$ ), testosterone $(\mathrm{T} ; t(40)=5.0, p<0.001)$, dihydrotestosterone (DHT; $t(19.7)=7.3, \quad p<0.001)$, and $3 \alpha$-androstanediol glucuronide $(3 \alpha$-diol; $t(26.1)=4.1, p<0.001)$ were significantly lower in middle-aged than young women, but sex hormone-binding globulin, and albumin (both $p>0.15$ ) levels were not significantly different between the age
Table I Baseline Serum Concentrations in Young $(N=17)$ and Middle-Aged $(N=25)$ Women in the Placebo Conditions, and Serum Concentrations in the Testosterone Condition Before and 30 min after a Nasal Testosterone Dose

\begin{tabular}{|c|c|c|c|c|c|c|c|c|}
\hline & \multirow{3}{*}{\multicolumn{2}{|c|}{$\begin{array}{c}\begin{array}{c}\text { Young } \\
\text { women }\end{array} \\
\text { Placebo } \\
\text { Baseline }\end{array}$}} & \multicolumn{6}{|c|}{ Middle-aged women } \\
\hline & & & \multirow{2}{*}{\multicolumn{2}{|c|}{$\begin{array}{l}\text { Placebo } \\
\text { Baseline }\end{array}$}} & \multicolumn{4}{|c|}{ Testosterone } \\
\hline & & & & & \multicolumn{2}{|c|}{ Baseline } & \multicolumn{2}{|c|}{$30 \mathrm{~min}$} \\
\hline & Mean & SEM & Mean & SEM & Mean & SEM & Mean & SEM \\
\hline $3 \alpha$-diol (pmol/l) & 6183.7 & 727.8 & 2774.8 & 412.0 & 2732.1 & 354.0 & 2736.4 & 352.3 \\
\hline Albumin $(g / l)$ & 43.1 & 0.9 & 42.4 & 0.6 & 42.3 & 0.5 & - & - \\
\hline DHEA-S (nmol/l) & 5847.1 & 536.9 & 3169.5 & 307.3 & 3173.9 & 311.9 & - & - \\
\hline DHT (pmol/l) & 605.1 & 41.7 & 265.2 & 20.9 & 279.8 & 36.9 & 367.9 & 40.1 \\
\hline Estradiol (pmol/l) & - & - & 186.5 & 33.2 & 144.8 & 18.3 & | 46.4 & 18.9 \\
\hline $\mathrm{SHBG}(\mathrm{nmol} / \mathrm{l})$ & 79.1 & 6.4 & 100.0 & 11.9 & 95.8 & 8.3 & - & - \\
\hline $\mathrm{T}(\mathrm{pmol} / \mathrm{l})$ & 964.8 & 58.3 & 637.2 & 37.5 & 672.7 & 52.7 & 3398.1 & 404.5 \\
\hline
\end{tabular}

Abbreviations: $3 \alpha$-diol, $3 \alpha$-androstanediol glucuronide; DHEA-S, dehydroepiandrosterone sulfate; DHT, dihydrotestosterone; SHBG, sex hormone-binding globulin; T, testosterone.

groups (see Table 1). The age groups did not significantly differ in state anxiety as measured with the STAI after scanning (mean \pm SEM; young: $35.5 \pm 2.0$; middle-aged: $33.1 \pm 1.6 ; p>0.3$ ), but the younger women were more accurate in matching the stimuli in both experimental conditions (emotion and control condition) than the middle-aged women (young: $98.2 \pm 0.9 \%$ correct, middleaged: $\quad 91.5 \pm 1.8 \% \quad$ correct; $\quad \mathrm{F}(1,35)=8.7, \quad p=0.006$ ). In addition, the young women were faster than the middle-aged women in all responses (young: $1460 \pm 59 \mathrm{~ms}$, middle-aged: $1748 \pm 92 \mathrm{~ms} ; \mathrm{F}(1,35)=5.6, p=0.024)$, but no significant group $\times$ task condition interactions were observed.

Imaging results. In the young women, the emotion condition yielded larger responses than the visuo-motor control condition in the amygdala, ventral visual stream (ranging from the primary visual cortex to the fusiform gyrus), middle temporal gyrus, inferior prefrontal gyrus, midbrain, supplementary motor area, premotor cortex, and occipital gyrus (see Figure 1a and Table 2). In middle-aged women, the ventral visual stream, inferior prefrontal gyrus, and supplementary motor area were also significantly activated. In addition, the neural response in the temporal pole was larger during the emotion than control condition, but no significant activation was observed in the amygdala (see Figure $1 \mathrm{~b}$ and Table 2). After lowering the statistical threshold, a non-significant activation within the amygdala was observed (peak voxel: [-20 -6 -13], $t(24)=2.6, p=0.008$, uncorrected), potentially indicating a weak or variable amygdala response.

Comparing neural responses between the young and middle-aged women revealed that amygdala reactivity was larger in the young than middle-aged women. In addition, 
a

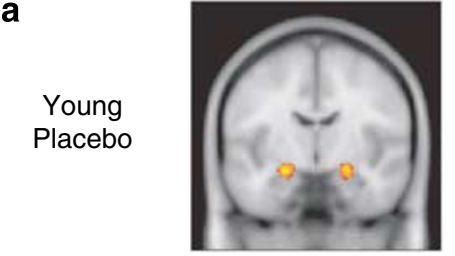

b

Middle-aged Placebo

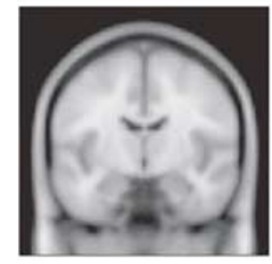

c

Middle-aged Testosterone

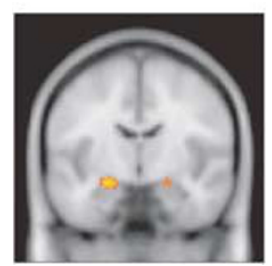

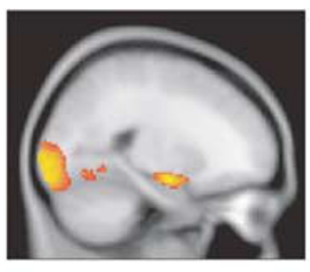
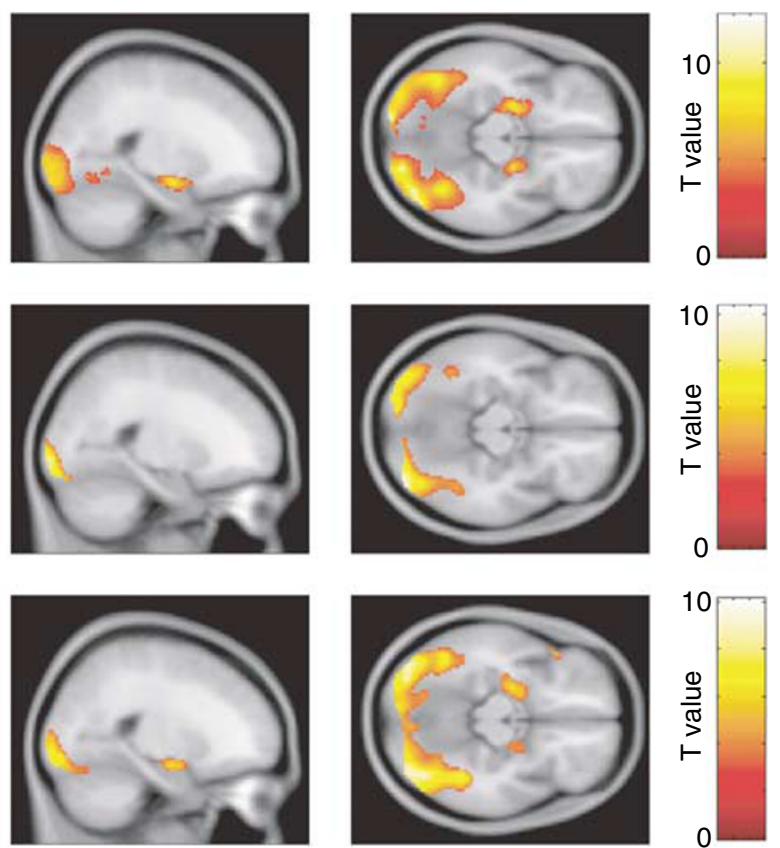

Figure I Amygdala reactivity to biologically salient stimuli in young and middle-aged women. (a) Significant amygdala activity in the placebo condition of young women $(\mathrm{N}=17)$. (b) No significant amygdala activity in the placebo condition of middle-aged women $(N=25)$. (c) Significant amygdala activity in middle-aged women after a single nasal administration of testosterone $(N=25)$. All figures show coronal, sagittal, and axial planes at the amygdala peak $([-22-8-1 \mathrm{I}])$ at a statistical threshold of $p<0.00 \mathrm{I}$, uncorrected, cluster $\geqslant 20$ voxels $(T(I 6) \geqslant 3.69$ in a; $T(24) \geqslant 3.47$ in $b$ and $c)$.

the neural response to emotional faces was larger in the young than middle-aged women in the ventral visual stream ranging from the extra-striate visual cortex to the fusiform gyrus. Conversely, the neural response to emotional faces was larger in the middle-aged than young women in the superior temporal gyrus, superior frontal gyrus, anterior cingulate cortex, superior frontal gyrus, middle frontal gyrus, and operculum (see Table 3). After correction for task performance by including reaction times as covariate, the bilateral superior frontal gyri and right superior temporal gyrus responses remained significantly larger in the middle-aged than young women. Importantly, the pattern of results with significantly larger amygdala and ventral visual stream responses in the young than middleaged women remained virtually identical. The differences in amygdala and superior frontal activity between the groups are therefore not related to differences in task performance.

To investigate whether these age differences in neural responses could be explained by differences in endogenous testosterone levels, a correlation analysis was performed. The testosterone levels of the young and middle-aged women during the placebo sessions were positively correlated with neural responses in the amygdala, fusiform gyrus, and insula across individuals (see Figure 2 and Table 4). Neural responses in the posterior cingulate cortex and superior frontal gyrus correlated negatively with testosterone levels (see Table 4). Although these correlations indicate that age differences in amygdala reactivity could be mediated by differences in testosterone levels, these associations are inherently confounded by age effects as testosterone levels were highly correlated with age $(\mathrm{r}(42)=-0.53, p<0.001)$. Inclusion of age as covariate did not alter the pattern of results in the amygdala and fusiform gyrus, but these positive correlations did not remain significant after correction for multiple comparisons. To investigate whether testosterone indeed mediates amygdala reactivity, a crossover placebo-controlled design was used.

Rapid Effects of Testosterone on Androgen Levels and Amygdala Reactivity in Middle-Aged Women

Serum concentrations, questionnaires, and behavioral performance. No significant differences were observed in all serum measures of the baseline samples of the two study days (ie T, DHT, $3 \alpha$-diol, DHEA-S, estradiol, progesterone, luteinizing hormone, sex hormone-binding globulin, and albumin; all paired $t$-tests $p>0.2$ ). The nasal testosterone dose increased the $\mathrm{T}$ (drug $\times$ time interactions: $\mathrm{F}(1,24)=48.1, \quad p<0.001)$ and DHT $(\mathrm{F}(1,23)=10.8$, $p=0.003)$ concentrations at $30 \mathrm{~min}$ after administration, but the $3 \alpha$-diol $(p>0.15)$ and estradiol $(p>0.3)$ levels were not significantly increased (see Table 1 ). The testosterone level that was reached is within the upper range for healthy women (Zumoff et al, 1995; Davison et al, 2005). Testosterone did not influence sexual lust, bodily arousal, and genital arousal as measured with the SAQ (drug $\times$ time interactions; $p>0.2$ ), state anxiety as measured with the STAI (mean \pm SEM; placebo: $33.1 \pm 1.6$; testosterone: $33.1 \pm 1.9 ; p>0.9$ ), and alertness (placebo: $322.4 \pm 22.6$; testosterone: $315.8 \pm 25.6 ; p>0.8$ ), contentedness (placebo: $240.3 \pm 9.9$; testosterone: $247.0 \pm 10.4 ; p>0.6$ ), and calmness (placebo: $110.7 \pm 5.0$; testosterone: $111.5 \pm 6.9 ; p>0.9$ ) as measured with the mood rating scale. In addition, testosterone did not significantly modulate response accuracy (percentage correct; placebo: emotion condition $89.8 \pm 2.4 \%$, control condition $93.2 \pm 1.8 \%$; testosterone: emotion condition $91.0 \pm 2.8 \%$, control condition 
Table 2 Main Effects of Task for the Young $(N=17)$ and MiddleAged $(N=25)$ Women in the Placebo Condition, and for MiddleAged Women after a Single Nasal Testosterone Dose (ie Matching Angry and Fearful Faces > Matching Scrambled Face Stimuli)

\begin{tabular}{|c|c|c|c|c|c|}
\hline & $x$ & $y$ & $\mathbf{z}$ & Cluster size & $\mathbf{Z}$ \\
\hline \multicolumn{6}{|l|}{ Young women: placebo condition } \\
\hline $\mathrm{R}$ inferior occipitotemporal cortex & 28 & -88 & -2 & 4096 & 6.1 \\
\hline$L$ inferior occipitotemporal cortex & -12 & -95 & -5 & 4186 & 6.0 \\
\hline $\mathrm{L}$ amygdala & -22 & -8 & -11 & 281 & 5.1 \\
\hline R amygdala & 22 & -8 & -11 & 150 & 4.8 \\
\hline $\mathrm{R}$ middle temporal gyrus & 46 & -56 & 16 & 78 & 4.6 \\
\hline$L$ inferior frontal gyrus & -42 & 9 & 27 & 532 & 4.5 \\
\hline $\mathrm{R}$ inferior frontal gyrus & 44 & 11 & 27 & 721 & 4.3 \\
\hline Midbrain & 4 & -35 & 2 & 157 & 4.3 \\
\hline Supplementary motor area & -2 & 11 & 35 & 208 & 4.2 \\
\hline R premotor cortex & 42 & 2 & 44 & 84 & 4.1 \\
\hline R occipital gyrus & 38 & -69 & 24 & 31 & 3.6 \\
\hline \multicolumn{6}{|l|}{ Middle-aged women: placebo condition } \\
\hline L inferior occipitotemporal cortex & -26 & -93 & । & 2262 & 6.3 \\
\hline R inferior occipitotemporal cortex & 22 & -95 & -2 & 2638 & 6.3 \\
\hline $\mathrm{R}$ inferior frontal gyrus & 48 & 22 & 21 & 501 & 5.1 \\
\hline$L$ inferior frontal gyrus & -53 & 17 & 27 & 263 & 4.7 \\
\hline Supplementary motor area & 2 & 12 & 47 & 368 & 4.6 \\
\hline $\mathrm{L}$ temporal pole & -30 & 5 & -19 & 49 & 3.9 \\
\hline \multicolumn{6}{|l|}{ Middle-aged women: testosterone condition } \\
\hline$L / R$ inferior occipitotemporal cortex & 18 & -89 & । & 7642 & 6.3 \\
\hline $\mathrm{R}$ inferior frontal gyrus & 48 & 9 & 33 & 1543 & 5.5 \\
\hline$L$ inferior frontal gyrus & -38 & 11 & 27 & 851 & 4.7 \\
\hline L amygdala & -22 & -8 & -11 & 224 & 4.6 \\
\hline L orbitofrontal cortex & -48 & 23 & -13 & 40 & 4.3 \\
\hline Medial superior frontal gyrus & 4 & 27 & 39 & 124 & 4.1 \\
\hline Precuneus & 0 & -58 & 49 & 50 & 3.9 \\
\hline R hippocampus & 22 & -22 & -6 & 49 & 3.9 \\
\hline $\mathrm{R}$ amygdala & 20 & -6 & -11 & 56 & 3.5 \\
\hline
\end{tabular}

Data are Talairach coordinates for cluster maxima at $p<0.001$ with a cluster size $\geqslant 20$ voxels; Cluster size in number of significant voxels.

$96.0 \pm 1.6 \%$; $p>0.4$ ) or reaction times (placebo: emotion condition $2237 \pm 102 \mathrm{~ms}$, control condition $1260 \pm 103 \mathrm{~ms}$; testosterone: emotion condition $2205 \pm 102 \mathrm{~ms}$, control condition $1245 \pm 113 \mathrm{~ms} ; p>0.7)$ during the behavioral task. These results indicate that the testosterone-induced differences in neural responses are unlikely explained by changes in mood states or behavioral performance.

Imaging results. In the testosterone session of the middleaged women, the emotion condition yielded larger responses than the visuo-motor control condition in the ventral visual stream (ranging from the primary visual cortex to the fusiform gyrus), inferior frontal gyrus, orbitofrontal cortex, medial superior frontal gyrus, precuneus, and hippocampus. Importantly, significant bilateral
Table 3 Effects of Age on Emotion Processing During the Placebo Conditions (ie Age $\times$ Task Interaction)

\begin{tabular}{|c|c|c|c|c|c|c|}
\hline & $x$ & $y$ & $\mathbf{z}$ & $\begin{array}{l}\text { Cluster } \\
\text { size }\end{array}$ & $\mathbf{Z}$ & SVC $p$ \\
\hline \multicolumn{7}{|l|}{ Young > middle-aged women } \\
\hline R inferior occipital cortex & 32 & -81 & 6 & 568 & 4.6 & \\
\hline $\begin{array}{l}L \text { inferior occipitotemporal } \\
\text { cortex }\end{array}$ & -22 & -83 & 4 & 753 & 4.5 & \\
\hline$L$ fusiform gyrus ${ }^{a}$ & -42 & -57 & -7 & 61 & 4.3 & 0.001 \\
\hline R fusiform gyrus ${ }^{a}$ & 36 & -57 & -6 & 73 & 3.8 & 0.006 \\
\hline $\mathrm{L}$ amygdala ${ }^{\mathrm{a}}$ & -24 & -6 & -13 & 46 & 3.7 & 0.004 \\
\hline L lingual gyrus & -22 & -58 & 0 & 47 & 3.5 & \\
\hline R amygdala ${ }^{a}$ & 28 & -12 & -11 & I & 3.2 & 0.017 \\
\hline \multicolumn{7}{|l|}{ Middle-aged > young women } \\
\hline R superior temporal gyrus & 67 & -19 & 12 & 82 & 4.4 & \\
\hline L superior frontal gyrus & -24 & 41 & 37 & 63 & 4.0 & \\
\hline Anterior cingulate cortex & -6 & 31 & 0 & 73 & 3.9 & \\
\hline R superior frontal gyrus & 16 & 20 & 48 & 158 & 3.9 & \\
\hline $\mathrm{R}$ middle frontal gyrus & 28 & 56 & -1 & 20 & 3.7 & \\
\hline L operculum & -65 & -26 & 25 & 25 & 3.5 & \\
\hline
\end{tabular}

Data are Talairach coordinates for cluster maxima at $p<0.00$ I with a cluster size $\geqslant 20$ voxels; Cluster size in number of voxels ( $p<0.001$, uncorrected); SVC $p$ is small volume corrected $p$-value.

aData from the search volume of the region of interest.
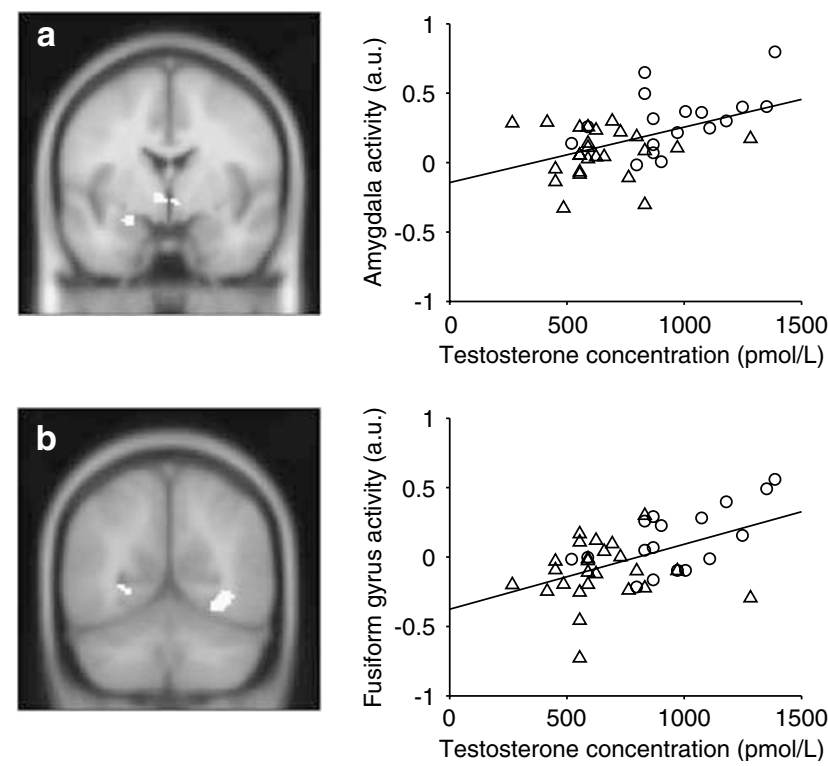

Figure 2 Baseline serum testosterone level correlates positively with amygdala $(\mathrm{a} ; y=-3)$ and fusiform gyrus $(\mathrm{b} ; \mathrm{y}=-58)$ activity across young (circles) and middle-aged (triangles) women in the placebo sessions. Left figures show coronal planes (left $=$ left) at the cluster maxima at a statistical threshold of $p<0.005$, uncorrected, for illustration.

amygdala activity was observed (see Figure 1c and Table 2). Direct comparisons showed that testosterone increased left amygdala reactivity in the middle-aged women (see Table 5). A similar effect was also observed in the right 
Table 4 Correlation Analysis Between Testosterone Levels and Neural Responses to Emotional Faces (ie Matching Angry and Fearful Faces > Matching Scrambled Face Stimuli) in the Placebo Session of the Young and Middle-Aged Women

\begin{tabular}{|c|c|c|c|c|c|c|}
\hline & $x$ & $y$ & $\mathbf{z}$ & Cluster size & $\mathbf{Z}$ & SVC $p$ \\
\hline \multicolumn{7}{|l|}{ Positive correlation } \\
\hline$L$ insula & -40 & -14 & -6 & 53 & 3.8 & \\
\hline R fusiform gyrus ${ }^{\mathrm{a}}$ & 32 & -55 & -6 & 26 & 3.2 & 0.041 \\
\hline $\mathrm{L}$ amygdala ${ }^{\mathrm{a}}$ & -24 & -3 & -13 & I & 3.3 & 0.015 \\
\hline \multicolumn{7}{|l|}{ Negative correlation } \\
\hline Posterior cingulate cortex & -8 & -33 & 35 & 145 & 3.8 & \\
\hline R superior frontal gyrus & 24 & 31 & 43 & 32 & 3.4 & \\
\hline
\end{tabular}

Table 5 Effects of a Single Nasal Testosterone Administration on Emotion Processing in Middle-Aged Women (ie Drug $\times$ Task Interaction), and Correlations with Serum Testosterone Levels in the Testosterone Condition Across Participants

\begin{tabular}{|c|c|c|c|c|c|c|}
\hline & $\mathbf{x}$ & y & $\mathbf{z}$ & Cluster size & $\mathbf{Z}$ & SVC $p$ \\
\hline \multicolumn{7}{|l|}{ Testosterone > placebo } \\
\hline L amygdala ${ }^{a}$ & -30 & -12 & -11 & 18 & 4.2 & 0.001 \\
\hline $\mathrm{R}$ inferior frontal gyrus & 52 & 27 & 5 & 55 & 4.0 & \\
\hline $\mathrm{L}$ middle temporal gyrus & -48 & 0 & -14 & 88 & 3.6 & \\
\hline $\mathrm{R}$ amygdala ${ }^{\mathrm{a}}$ & 24 & -12 & -13 & - & 2.4 & 0.109 \\
\hline \multicolumn{7}{|l|}{ Placebo $>$ testosterone } \\
\hline Precuneus & -10 & -32 & 50 & 22 & 3.4 & \\
\hline \multicolumn{7}{|c|}{ Testosterone condition: positive correlation with testosterone level } \\
\hline L middle frontal gyrus & -30 & 21 & 30 & 86 & 4.6 & \\
\hline R superior frontal gyrus & 12 & 33 & 48 & 31 & 3.8 & \\
\hline L supramarginal gyrus & -50 & -49 & 28 & 80 & 3.8 & \\
\hline R precuneus & 8 & -60 & 42 & 25 & 3.4 & \\
\hline \multicolumn{7}{|c|}{ Testosterone condition: negative correlation with testosterone level } \\
\hline R occipital gyrus & 10 & -91 & -10 & 34 & 3.7 & \\
\hline R orbitofrontal cortex & 28 & 22 & -22 & 27 & 3.5 & \\
\hline L orbitofrontal cortex & -27 & 19 & -17 & 46 & 3.5 & \\
\hline
\end{tabular}

Data are Talairach coordinates for cluster maxima at $p<0.00$ I with a cluster size $\geqslant 20$ voxels; Cluster size in number of voxels ( $p<0.00$ I, uncorrected); SVC $p$ is small volume corrected $p$-value.

${ }^{\mathrm{a}}$ Data from the search volume of the region of interest.

amygdala, although at a less conservative statistical threshold ([24 -12 -13], $t(24)=2.6, p=0.008$, uncorrected). In addition, testosterone increased the neural response to emotional faces in the inferior frontal and middle temporal gyri. Conversely, testosterone reduced the neural response to emotional faces in the precuneus (see Table 5).
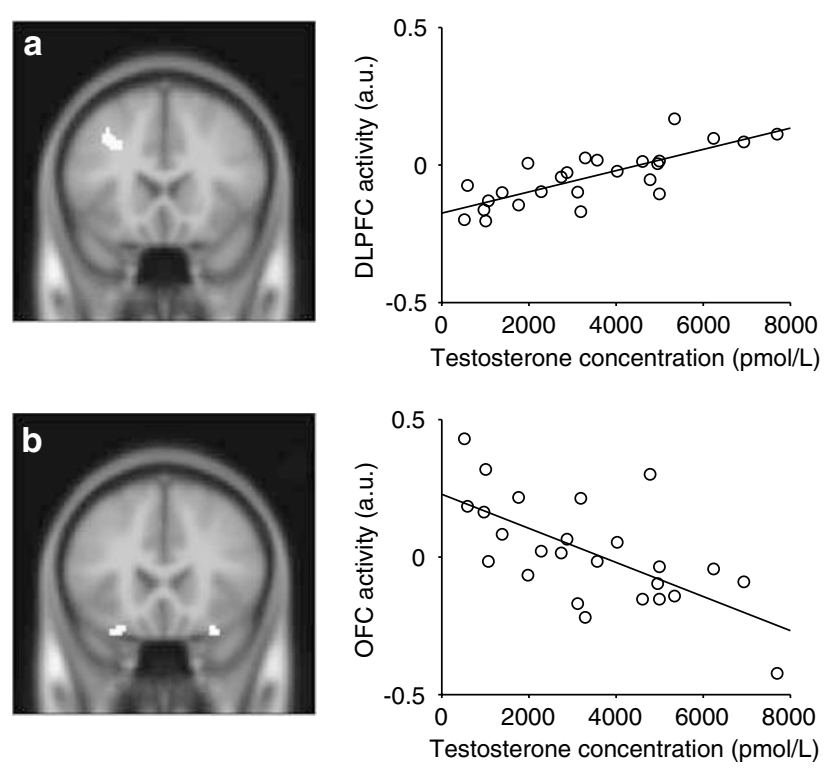

Figure 3 Serum testosterone level after a single nasal testosterone dose correlates positively with left dorsolateral prefrontal cortex (a; DLPFC, BA $9, y=21$ ) and negatively with orbitofrontal cortex (b; OFC, BA 47, $y=22$ ) activity across middle-aged women. Left figures show coronal planes (left $=$ left) at the cluster maxima at a statistical threshold of $p<0.00$ I, uncorrected, cluster $\geqslant 20$ voxels.

A correlation analysis was performed to identify whether the enhanced testosterone levels were related to neural responses across individuals. Testosterone levels correlated positively with neural responses in the middle frontal gyrus (BA 9), superior frontal gyrus, supramarginal gyrus, and precuneus. Testosterone levels correlated negatively with responses in the orbitofrontal cortex (BA 47) and occipital gyrus (see Table 5 and Figure 3). Although testosterone reliably increased amygdala reactivity within subjects, no significant correlations with amygdala reactivity were observed across individuals, indicating individual differences in sensitivity to high testosterone levels.

To investigate whether testosterone increased amygdala reactivity of the middle-aged women to a level comparable to the young women, the data from the amygdala ROIs were extracted and averaged. The ROI analyses confirmed that amygdala reactivity was higher in the young than middleaged women (left: $t(40)=3.1, p=0.004$, effect size: $r=0.44$; right: $t(40)=2.1, p=0.043, r=0.31)$, and that testosterone increased left amygdala reactivity in the middle-aged women (left: $t(24)=2.2, p=0.035, r=0.41$; right: $t(24)=$ $1.3, p=0.19$, NS, $r=0.27)$. Importantly, testosterone increased amygdala reactivity in middle-aged women to a level comparable to that of young women (left: $t(40)=1.2$, $p=0.25, \quad$ NS, $r=0.18$; right: $\mathrm{t}(40)=1.1, p=0.26, \quad \mathrm{NS}$, $r=0.18$; see Figure 4 ).

\section{DISCUSSION}

The present study investigated whether androgens influence amygdala reactivity in healthy, naturally cycling women. The results show that the age-related decline in androgen levels is associated with a decrease in amygdala reactivity. 


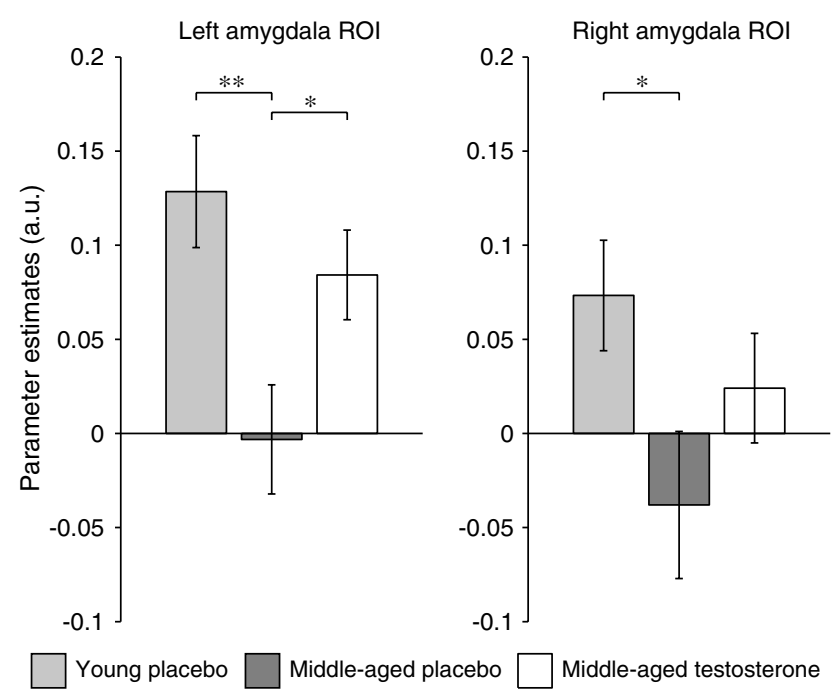

Figure 4 Testosterone increases amygdala reactivity to biologically salient stimuli in middle-aged women $(N=25)$ to a level comparable to young women $(N=17)$. The figure shows the mean $( \pm S E M)$ amygdala response averaged across the amygdala $\mathrm{ROI} .{ }^{*} * 0.0 \mathrm{l}$, ${ }^{*} p<0.05$.

A double-blind, placebo-controlled, crossover study was applied to investigate whether exogenous testosterone modulates amygdala reactivity in premenopausal middleaged women, who have about half their early adulthood androgen levels. The results show that a single nasal testosterone administration rapidly (within $45 \mathrm{~min}$ ) increases amygdala reactivity in the middle-aged women to a young adulthood level.

The amygdala is part of a larger emotion circuitry, which is important for the identification of the emotional significance of stimuli, the generation of an affective response, and emotion regulation (Phillips et al, 2003). The endogenous testosterone levels were positively related to neural responses in the fusiform gyrus. This inferior temporal brain region is preferentially involved in face processing (Kanwisher et al, 1997) and in interaction with the amygdala important for emotional face perception (Haxby et al, 2002). The amygdala projects back to all levels of the ventral visual stream (Amaral et al, 2003), influencing emotional face perception (Vuilleumier et al, 2004). This suggests that the increased fusiform gyrus responses are a potential consequence of increased amygdala responses, indicating that testosterone may influence the perception of emotional stimuli. The enhanced testosterone levels were positively correlated with superior frontal cortex responses, and negatively with orbitofrontal cortex responses. Whereas the superior frontal regions are implicated in the controlled regulation of affect, the inferior frontal regions are thought to regulate emotional responses automatically via direct connections to the amygdala (Stefanacci and Amaral, 2002; Phillips et al, 2003; Ochsner and Gross, 2005; Stein et al, 2007). This suggests that the regulatory function of the orbitofrontal cortex decreases with increasing testosterone levels, potentially contributing to the observed amygdala disinhibition. In addition, the increased superior frontal cortex activity may reflect compensatory effort to regulate emotional responses.
The age-related changes in neural responses to faces between young adulthood and midlife reported in the present study are similar to the changes that have been reported previously in elderly. While amygdala and fusiform gyrus activity decreases, prefrontal activity increases in healthy older men and women ( $>60$ years) in comparison to young adults ( $<30$ years) (Iidaka et al, 2002; Gunning-Dixon et al, 2003; Mather et al, 2004; Tessitore et al, 2005). However, to our knowledge, amygdala reactivity during midlife has not been investigated previously. The results of the present study show that amygdala reactivity in naturally cycling women already decreases before menopause, when androgen levels have declined to half the early adulthood level. The correlation between decreasing endogenous testosterone levels and decreasing neural responses, together with the testosterone-induced amygdala reactivity increase, suggest that endogenous decreases in androgen levels may contribute to age-related decreases in amygdala reactivity.

Whereas the endogenous testosterone levels correlated positively with amygdala reactivity across individuals, the enhanced testosterone levels did not, even though testosterone reliably increased amygdala reactivity within subjects. This suggests that individual differences in amygdala sensitivity to high testosterone levels may exist. Furthermore, although the testosterone levels in the middle-aged women increased beyond the endogenous level of healthy young women, amygdala reactivity was similar. A recent study showed that a single testosterone administration also increases amygdala responses in healthy young women (Hermans et al, 2007). This suggests that testosterone increases amygdala reactivity within individuals regardless of baseline responses, but the absolute range of testosterone-potentiated amygdala responses may differ across individuals and age.

The rapid effect of nasal testosterone could be mediated by the rapid absorption of testosterone into the systemic circulation, but the nasal mucosa also provides a potential for pharmaceutical compounds to be available to the central nervous system directly (Hussain, 1998). Animal studies suggest that the timing of the testosterone-induced effect (within $45 \mathrm{~min}$ ) indicates a nongenomic mechanism of action, potentially mediated by its rapid conversion to estradiol or its neuroactive steroid $3 \alpha$-androstanediol. Studies in male mice have shown that changes in sexual behavior can be mediated by changes in estrogen levels, due to rapid changes in aromatase activity (Balthazart et al, 2006; Taziaux et al, 2007). In addition, testosterone also has anxiolytic properties, and its rapid anxiolytic action appears mediated by conversion to $3 \alpha$-androstanediol, which potentiates inhibitory neurotransmission by modulation of the $\mathrm{GABA}_{\mathrm{A}}$ receptor (Aikey et al, 2002). This latter action may be similar to that of progesterone, which increases amygdala reactivity in healthy young women (van Wingen et al, 2007). Animal studies have demonstrated that the acute effects of progesterone are mediated by its neuroactive metabolite allopregnanolone that also exerts its action by binding to the $\mathrm{GABA}_{\mathrm{A}}$ receptor (Bitran et al, 1991, 1995), suggesting a common pathway by which neuroactive steroids could modulate amygdala function.

Testosterone did not influence self-reported sexual arousal, state anxiety, and mood in this study, which is in 
line with previous studies (van Honk et al, 2001). However, testosterone may change subjective arousal after appropriate stimulation. For example, whereas testosterone increases physiological but not subjective arousal after a single exposure to erotic movies, repeated exposure to erotic movies also increases subjective sexual arousal (Tuiten et al, 2000, 2002). In addition, prolonged exposure to testosterone, or the prolonged withdrawal from testosterone, does influence the subjective experience. Mood and sexual function worsen after oophorectomy, when gonadal steroid hormone concentrations are chronically decreased. Moreover, subsequent testosterone treatment improves mood and sexual function in those women (Sherwin and Gelfand, 1985; Sherwin et al, 1985; Shifren et al, 2000). This suggests that although testosterone did not modulate selfreport measures in this study, it may do so after appropriate stimulation or repeated administration.

This study shows that testosterone modulates amygdala activity in women, and animal research suggests that it may have a similar effect in men (Dimeo and Wood, 2006), for whom testosterone also modulates mood and sexual function (Schmidt et al, 2004). Also the female gonadal hormones progesterone (van Wingen et al, 2007) and presumably estradiol (Goldstein et al, 2005) modulate amygdala activity in women. The influence of these hormones in this study is likely limited, because the women were investigated during their follicular phase when progesterone and estradiol concentrations are lowest and similar to those in men. In addition, testosterone levels are also relatively low during the follicular phase, and increase during midcycle (Judd and Yen, 1973; Sinha-Hikim et al, 1998). Nevertheless, the sex hormones may interactively modulate amygdala activity. Furthermore, the amygdala is a sexual dimorphic brain structure with a relatively larger volume in men than women (Goldstein et al, 2001). The sex differences in amygdala structure and circulating hormone levels could mediate the sex differences in amygdala responsivity (Hamann, 2005; Cahill, 2006), and may thereby contribute to sex differences in the prevalence of psychiatric disorders (Kessler et al, 1994).

\section{DISCLOSURE/CONFLICTS OF INTEREST}

This work was supported by a research grant from $\mathrm{M}$ et $\mathrm{P}$ Pharma AG (Stans, Switzerland), the manufacturer of the intranasal testosterone gel used in this study. Dr Mattern is Chief Technology Officer at M et P Pharma AG. The other authors have no potential conflict of interest.

\section{REFERENCES}

Aikey JL, Nyby JG, Anmuth DM, James PJ (2002). Testosterone rapidly reduces anxiety in male house mice (Mus musculus). Horm Behav 42: 448-460.

Amaral DG, Behniea H, Kelly JL (2003). Topographic organization of projections from the amygdala to the visual cortex in the macaque monkey. Neuroscience 118: 1099-1120.

Amunts K, Kedo O, Kindler M, Pieperhoff P, Mohlberg H, Shah NJ et al (2005). Cytoarchitectonic mapping of the human amygdala, hippocampal region and entorhinal cortex: intersubject variability and probability maps. Anat Embryol (Berl) 210: 343-352.
Balthazart J, Baillien M, Ball GF (2006). Rapid control of brain aromatase activity by glutamatergic inputs. Endocrinology 147: 359-366.

Bialy M, Sachs BD (2002). Androgen implants in medial amygdala briefly maintain non-contact erection in castrated male rats. Horm Behav 42: 345-355.

Bitran D, Hilvers RJ, Kellogg CK (1991). Anxiolytic effects of 3 alpha-hydroxy-5 alpha[beta]-pregnan-20-one: endogenous metabolites of progesterone that are active at the GABAA receptor. Brain Res 561: 157-161.

Bitran D, Shiekh M, Mcleod M (1995). Anxiolytic effect of progesterone is mediated by the neurosteroid allopregnanolone at brain gaba(a) receptors. J Neuroendocrinol 7: 171-177.

Bond A, Lader M (1974). The use of analogue scales in rating subjective feelings. Br J Med Psychol 47: 211-218.

Brett M, Anton J, Valbregue R, Poline J (2002). Regions of interest analysis using an SPM toolbox [abstract]. Neuroimage 16(2 Suppl 1): 497.

Cahill L (2006). Why sex matters for neuroscience. Nat Rev Neurosci 7: 477-484.

Cohen J (1992). A power primer. Psychol Bull 112: 155-159.

Davison SL, Bell R, Donath S, Montalto JG, Davis SR (2005). Androgen levels in adult females: changes with age, menopause, and oophorectomy. J Clin Endocrinol Metab 90: 3847-3853.

Dimeo AN, Wood RI (2006). ICV testosterone induces Fos in male Syrian hamster brain. Psychoneuroendocrinology 31: 237-249.

Drevets WC (2003). Neuroimaging abnormalities in the amygdala in mood disorders. Ann NY Acad Sci 985: 420-444.

Eickhoff SB, Stephan KE, Mohlberg H, Grefkes C, Fink GR, Amunts $\mathrm{K}$ et al (2005). A new SPM toolbox for combining probabilistic cytoarchitectonic maps and functional imaging data. Neuroimage 25: 1325-1335.

Everitt BJ (1990). Sexual motivation: a neural and behavioural analysis of the mechanisms underlying appetitive and copulatory responses of male rats. Neurosci Biobehav Rev 14: 217-232.

Friston KJ, Holmes AP, Worsley KJ, Poline JB, Frith CD, Frackowiak RSJ (1995). Statistical parametric maps in functional imaging: a general linear approach. Hum Brain Mapp 2: 189-210.

Friston KJ, Penny W, Phillips C, Kiebel S, Hinton G, Ashburner J (2002). Classical and Bayesian inference in neuroimaging: theory. Neuroimage 16: 465-483.

Gizewski ER, Krause E, Karama S, Baars A, Senf W, Forsting M (2006). There are differences in cerebral activation between females in distinct menstrual phases during viewing of erotic stimuli: a fMRI Study. Exp Brain Res 174: 101-108.

Goldstat R, Briganti E, Tran J, Wolfe R, Davis SR (2003). Transdermal testosterone therapy improves well-being, mood, and sexual function in premenopausal women. Menopause 10: 390-398.

Goldstein JM, Seidman LJ, Horton NJ, Makris N, Kennedy DN, Caviness Jr VS et al (2001). Normal sexual dimorphism of the adult human brain assessed by in vivo magnetic resonance imaging. Cereb Cortex 11: 490-497.

Goldstein JM, Jerram M, Poldrack R, Ahern T, Kennedy DN, Seidman LJ et al (2005). Hormonal cycle modulates arousal circuitry in women using functional magnetic resonance imaging. J Neurosci 25: 9309-9316.

Gunning-Dixon FM, Gur RC, Perkins AC, Schroeder L, Turner T, Turetsky BI et al (2003). Age-related differences in brain activation during emotional face processing. Neurobiol Aging 24: 285-295.

Hamann S, Herman RA, Nolan CL, Wallen K (2004). Men and women differ in amygdala response to visual sexual stimuli. Nat Neurosci 7: 411-416.

Hamann S (2005). Sex differences in the responses of the human amygdala. Neuroscientist 11: 288-293. 
Hariri AR, Mattay VS, Tessitore A, Fera F, Smith WG, Weinberger DR (2002). Dextroamphetamine modulates the response of the human amygdala. Neuropsychopharmacology 27: 1036-1040.

Haxby JV, Hoffman EA, Gobbini MI (2002). Human neural systems for face recognition and social communication. Biol Psychiatry 51: 59-67.

Hayes R, Dennerstein L (2005). The impact of aging on sexual function and sexual dysfunction in women: a review of population-based studies. J Sex Med 2: 317-330.

Hayes RD, Dennerstein L, Bennett CM, Koochaki PE, Leiblum SR, Graziottin A (2007). Relationship between hypoactive sexual desire disorder and aging. Fertil Steril 87: 107-112.

Henderson AS, Jorm AF, Korten AE, Jacomb P, Christensen H, Rodgers B (1998). Symptoms of depression and anxiety during adult life: evidence for a decline in prevalence with age. Psychol Med 28: 1321-1328.

Hermans EJ, Ramsey NF, Honk JV (2007). Exogenous testosterone enhances responsiveness to social threat in the neural circuitry of social aggression in humans. Biol Psychiatry: doi:10.1016/ j.biopsych.2007.1005.1013.

Hussain AA (1998). Intranasal drug delivery. Adv Drug Deliv Rev 29: 39-49.

Iidaka T, Okada T, Murata T, Omori M, Kosaka H, Sadato N et al (2002). Age-related differences in the medial temporal lobe responses to emotional faces as revealed by fMRI. Hippocampus 12: $352-362$.

James PJ, Nyby JG (2002). Testosterone rapidly affects the expression of copulatory behavior in house mice (Mus musculus). Physiol Behav 75: 287-294.

Judd HL, Yen SSC (1973). Serum androstenedione and testosterone levels during menstrual-cycle. J Clin Endocrinol Metab 36: 475-481.

Kanwisher N, McDermott J, Chun MM (1997). The fusiform face area: a module in human extrastriate cortex specialized for face perception. J Neurosci 17: 4302-4311.

Karama S, Lecours AR, Leroux JM, Bourgouin P, Beaudoin G, Joubert $S$ et al (2002). Areas of brain activation in males and females during viewing of erotic film excerpts. Hum Brain Mapp 16: 1-13.

Kendrick KM, Drewett RF (1979). Testosterone reduces refractory period of stria terminalis neurons in the rat brain. Science 204: 877-879.

Kendrick KM (1981). Effect of testosterone and the oestrous cycle on neuronal refractory periods and firing rates of stria terminalis neurones in the female rat. Exp Brain Res 44: 331-336.

Kessler RC, McGonagle KA, Zhao S, Nelson CB, Hughes M, Eshleman $S$ et al (1994). Lifetime and 12-month prevalence of DSM-III-R psychiatric disorders in the United States: results from the National Comorbidity Survey. Arch Gen Psychiatry 51: $8-19$.

Kessler RC, Berglund P, Demler O, Jin R, Koretz D, Merikangas KR et al (2003). The epidemiology of major depressive disorder: results from the National Comorbidity Survey Replication (NCS-R). JAMA 289: 3095-3105.

Mather M, Canli T, English T, Whitfield S, Wais P, Ochsner K et al (2004). Amygdala responses to emotionally valenced stimuli in older and younger adults. Psychol Sci 15: 259-263.

Ochsner KN, Gross JJ (2005). The cognitive control of emotion. Trends Cogn Sci 9: 242-249.

Paulus MP, Feinstein JS, Castillo G, Simmons AN, Stein MB (2005). Dose-dependent decrease of activation in bilateral amygdala and insula by lorazepam during emotion processing. Arch Gen Psychiatry 62: 282-288.

Phillips ML, Drevets WC, Rauch SL, Lane R (2003). Neurobiology of emotion perception I: the neural basis of normal emotion perception. Biol Psychiatry 54: 504-514.

Rauch SL, Shin LM, Wright CI (2003). Neuroimaging studies of amygdala function in anxiety disorders. Ann NY Acad Sci 985: $389-410$
Schmidt PJ, Berlin KL, Danaceau MA, Neeren A, Haq NA, Roca CA et al (2004). The effects of pharmacologically induced hypogonadism on mood in healthy men. Arch Gen Psychiatry 61: 997-1004.

Sherwin BB, Gelfand MM (1985). Sex steroids and affect in the surgical menopause: a double-blind, cross-over study. Psychoneuroendocrinology 10: 325-335.

Sherwin BB, Gelfand MM, Brender W (1985). Androgen enhances sexual motivation in females: a prospective, crossover study of sex steroid administration in the surgical menopause. Psychosom Med 47: 339-351.

Shifren JL, Braunstein GD, Simon JA, Casson PR, Buster JE, Redmond GP et al (2000). Transdermal testosterone treatment in women with impaired sexual function after oophorectomy. $\mathrm{N}$ Engl J Med 343: 682-688.

Sinha-Hikim I, Arver S, Beall G, Shen R, Guerrero M, Sattler F et al (1998). The use of a sensitive equilibrium dialysis method for the measurement of free testosterone levels in healthy, cycling women and in human immunodeficiency virus-infected women. J Clin Endocrinol Metab 83: 1312-1318.

Spielberger CD, Gorsuch RL, Lushene RE (1970). STAI Manual for the State Trait Anxiety Inventory. Consulting Psychologists Press: Palo Alto.

Stefanacci L, Amaral DG (2002). Some observations on cortical inputs to the macaque monkey amygdala: an anterograde tracing study. J Comp Neurol 451: 301-323.

Stein JL, Wiedholz LM, Bassett DS, Weinberger DR, Zink CF, Mattay VS et al (2007). A validated network of effective amygdala connectivity. Neuroimage 36: 736-745.

Taziaux M, Keller M, Bakker J, Balthazart J (2007). Sexual behavior activity tracks rapid changes in brain estrogen concentrations. J Neurosci 27: 6563-6572.

Tessitore A, Hariri AR, Fera F, Smith WG, Das S, Weinberger DR et al (2005). Functional changes in the activity of brain regions underlying emotion processing in the elderly. Psychiatry Res 139: 9-18.

Tuiten A, Van Honk J, Koppeschaar H, Bernaards C, Thijssen J, Verbaten R (2000). Time course of effects of testosterone administration on sexual arousal in women. Arch Gen Psychiatry 57: 149-153.

Tuiten A, van Honk J, Verbaten R, Laan E, Everaerd W, Stam H (2002). Can sublingual testosterone increase subjective and physiological measures of laboratory-induced sexual arousal? Arch Gen Psychiatry 59: 465-466.

van Honk J, Tuiten A, Hermans E, Putman P, Koppeschaar H, Thijssen J et al (2001). A single administration of testosterone induces cardiac accelerative responses to angry faces in healthy young women. Behav Neurosci 115: 238-242.

van Wingen GA, van Broekhoven F, Verkes RJ, Petersson KM, Bäckström T, Buitelaar JK et al (2007). Progesterone selectively increases amygdala reactivity in women. Mol Psychiatry: doi:10.1038/sj.mp.4002030.

Vuilleumier P, Richardson MP, Armony JL, Driver J, Dolan RJ (2004). Distant influences of amygdala lesion on visual cortical activation during emotional face processing. Nat Neurosci 7: 1271-1278.

Wood RI, Newman SW (1995). The medial amygdaloid nucleus and medial preoptic area mediate steroidal control of sexual behavior in the male Syrian hamster. Horm Behav 29: 338-353.

Worsley KJ, Marrett S, Neelin P, Vandal AC, Friston KJ, Evans AC (1996). A unified statistical approach for determining significant signals in images of cerebral activation. Hum Brain Mapp 4: $58-73$.

Zumoff B, Strain GW, Miller LK, Rosner W (1995). Twenty-fourhour mean plasma testosterone concentration declines with age in normal premenopausal women. J Clin Endocrinol Metab 80: $1429-1430$ 